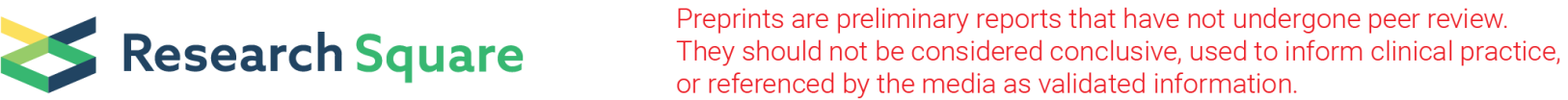

\section{Significance of The Neutrophil-To-Lymphocyte Ratio in Predicting the Response of Neoadjuvant Chemotherapy in Osteosarcoma: A Multicenter Retrospective Study}

\author{
Tang Haijun \\ Minzu Affiliated Hospital of Guangxi Medical University \\ Liu Dehuai \\ Minzu Affiliated Hospital of Guangxi Medical University \\ Lu Jili \\ People's Hospital of Baise \\ He Juliang \\ Affiliated Tumor Hospital of Guangxi Medical University \\ Ji Shuyu
}

The First Affiliated Hospital of Guangxi Medical University

\section{Liao Shijie}

The First Affiliated Hospital of Guangxi Medical University

\section{Wei Qingjun}

The First Affiliated Hospital of Guangxi Medical University

\section{Lu Shenglin}

Minzu Affiliated Hospital of Guangxi Medical University

\section{Yun Liu ( $\nabla 275207660 @ q q . c o m$ )}

The First Affiliated Hospital of Guangxi Medical University

\section{Research Article}

Keywords: neutrophil-to-lymphocyte, neoadjuvant chemotherapy, osteosarcoma

Posted Date: August 6th, 2021

DOI: https://doi.org/10.21203/rs.3.rs-707519/v1

License: (c) (1) This work is licensed under a Creative Commons Attribution 4.0 International License. Read Full License 


\section{Abstract \\ Background}

At present, there is no validated predictive factor for early efficacy of neoadjuvant hemotherapy (NACT) in osteosarcoma. The purpose of this study was to investigate the significance of the neutrophil-tolymphocyte ratio (NLR) in predicting the response of NACT in osteosarcoma.

\section{Methods}

Pathological complete response (pCR) was used to assess the efficacy of NACT. Receiver Operating Characteristic (ROC) curve and Youden index (sensitivity + specificity-1) were used to determine the optimal cut-off values of NLR. Univariate and multivariate analyses by logistic regression model were conducted to confirm the independent factors affecting the efficacy of NACT.

\section{Results}

The optimal cutoff value of NLR was 2.36 (sensitivity, 80.0\%; specificity, 71.3\%). Univariate analysis revealed that the smaller tumor volum, lower stage, lower NLR and lower PLR were more likely to achieve pCR. Multivariate analyses confirmed that NLR before treatment was an independent risk factor for pCR. Compared to patients with a high NLR, those with a low NLR showed more than 2-fold higher chance to achieve pCR (OR 2.82, 95\% Cl 1.36-5.17, p = 0.02).

\section{Conclusion}

NLR is a novel and effective factor predicting the response to NACT in osteosarcoma patients. Patients with higher NLR showed a lower percentage of pCR after NACT.

\section{Introduction}

Osteosarcoma, which is originated from mesenchymal tissue and mainly located in the long bones, is one of the most common primary malignant tumor in children and adolescent [1]. Before 1970s, the main treatment method for this disease was amputation associated with postoperative chemotherapy. However, the prognosis of patients treated with this combination was extremly poor and the 5-year survival rate was approximately 42\% [2]. In 1970, Cortes [3] and Rosen [4] subsequently proposed preoperative chemotherapy which is also known as the neo-adjuvant hemotherapy (NACT). Since then, the survival rate has raised to $60 \%-70 \%$ and limb salvage is possible for most patients [5].

According to the recommendation by National Compre-hensive Cancer Network (NCCN), the curative effect of chemotherapy is a key index assessing whether the limb salvage is feasibility [6]. Hence, it is 
very important to predict the efficacy of NACT before surgery. Recently, pathological complete response (pCR) is regarded as a gold standard to estimate the efficacy of NACT [7]. However, pCR is obtained based on postoperative tumor specimens, so it cann't be validated as predictive factor for early efficacy of NACT. Additionally, some radiological parameters, such as tumor volume, apparent diffusion coefficient (ADC) on MRI, standardized uptake values (SUV) on ${ }^{18} \mathrm{~F}$-FDG PET and ect are also proposed as predictive index $[8,9]$. Although these parameters can be measured before surgery and possess partly satisfied reliability, the error in measurement, the specificity and sensitivity, and the cost are still need to be improved. Therefore, it is significant important to search some new, reliable, and inexpensive parameters.

At present, it has been demostrated that system inflammation response (SIR) plays a key role in tumorigenesis, Metastasis, and even drug resistance [10]. The neutrophil-to-lymphocyte ratio (NLR) and platelet-to-lymphocyte ratio (PLR) are two important index to reflect SIR [11], which have also been studied for predicting the PCR in various solid tumor such as breast and esophageal cancer [12, 13]. It is generally true that the lower NLR and PLR are associated with weaker inflammation, which leads to better pCR and prognosis. However, the potential contribution of these two indicators to NACT response has been not reported.

Thus, we conducted this multicenter retrospective study to determine whether NLR or PLR could be used to predict the pCR after NACT in osteosarcoma. If it exactly do, it can be regarded as a parameter to provide consult for formulating chemotherapy and sugical regimen. To the best of our knowledge, it is the first time we report the association of NACT response with NLR and PLR in osteosarcoma.

\section{Patients And Methods}

\subsection{Patients}

This multicenter study was conducted in three medical institutions. Medical records of patients with osteosarcoma was reviewed retrospectively from September 2008 to December 2018. Inclusion criterias was formulated as follows: (1) Patients were diagnosed as osteosarcoma via postoperative histopathology; (2) Patients received standard neoadjuvant chemotherapy (NACT) before surgery; (3) Patient owned blood routine examination before NACT; (4) Patient's surgical methods were tumor resection or amputation so that pathological complete response ( $\mathrm{pCR}$ ) was available; (5) Lesion located in extremities. Exclusion criterias: (1) Patient with infection, fever, or some blood disease; (2) Recurrent patients; (3) Patients with incompleted medical record.

Clinical and pathological stage of the tumor were defined via Ennecking's classifications [14]. The informed consents were obtained from all patients and this study was supported by Ethical Association of our institution.

\subsection{Blood samples and data review}


Blood samples were obtained when patient was hospitalised to start first cycle of chemotherapy. NLR was calculated via peripheral blood routine examination and defined as the absolute number of neutrophil divided by the lymphocyte counts. The same formula was applied in platelet-to-lymphocyte Ratio (PLR). Other parameters, including, white blood cell count (WBC), percentage of monocytes, erythrocyte sedimentation rate (ESR), C-reactive protein (CRP) and alkaline phosphatase (ALP) was also reviewed. Additionally, some clinical parameters, such as age, gender, location of lesion, size of tumor on imaging, and tumor subtype were corrected and analysed.

\subsection{Regimens and efficacy assessment for NACT}

All patient received their first-line NACT and the detail regimens were AP: Doxorubicin $45 \mathrm{mg} / \mathrm{m}^{2}+$ Cisplatin $75 \sim 100 \mathrm{mg} / \mathrm{m}^{2}$ or MAP: High dose methotrexate $8 \sim 12 \mathrm{~g} / \mathrm{m}^{2}+$ Doxorubicin $45 \mathrm{mg} / \mathrm{m}^{2}+$ Cisplatin $75 \sim 100 \mathrm{mg} / \mathrm{m}^{2}$.

Pathological complete response (pCR), which was defined as study endpoint in this study, was obtained after time of sugery and used to assess the efficacy of NACT. It was defined as good response as well as efficacy when the percentage of tumor necrosis was $90 \%$ or more, while less than $90 \%$ necrosis indicated poor response and efficacy.

\subsection{Statistical analysis}

The means \pm standard error and median were used to present measurement data which obeyed normal distribution and skewed distribution, respectively. Receiver Operating Characteristic (ROC) curve and Youden index (sensitivity + specificity-1) were used to determine the optimal cut-off values of NLR for its absence in the recent reported literature.

We used the chi-squared test and Fisher's exact test to evaluate the association between NLR, PLR, other important clinical parameter, and PCR. Univariate and multivariate analyses by logistic regression model were conducted to confirm the independent factors that predict PCR. Statistically significant was considered when $p<0.05$. All data was assembled using Excel 2007 and data analyses were performed by SPSS 22.0 software.

\section{Result}

\subsection{Baseline data of patients and tumor}

403 patients who diagosed as osteosarcoma histopathologically were identified. Ultimately, 96 patients met the Inclusion/Exclusion criterias and were included in this study. The baseline data was shown in Tble 1. Among these 96 patients, 54 were male and the median age at diagnosis was 17 years (range 745 years). Eighty-four (87.5\%) tumor were located in proximal tibia and distal femur while 12 in other region such as proximal humerus, proximal fibula and distal tibia. The median tumor size measured by MRI was $195.37 \pm 8.74 \mathrm{~cm}^{3}$. According to Enneking surgical staging criteria, 28 patients was regarded as stage $\otimes, 46$ as stage $\otimes$, and 22 as stage $\otimes$, respectively. 


\subsection{The optimal cutoff value of NLR and PLR}

As Fig. 1 shows, when pCR was defined as an end point, the areas under the curve (AUC) for NLR and PLR were $0.793(p=0.001)$ and $0.659(p=0.069)$, respectively. The optimal cutoff values of NLR and PLR were 2.36 (sensitivity, 80.0\%; specificity, 71.3\%) and 115 (sensitivity, 83.3\%; specificity, 55.6\%), respectively. Subsequently, according to the optimal cutoff values of NLR, patients were divided into two groups: low NLR group (LNLR <2.36), 50 patients and high NLR group (HNLR $\geq 2.36), 46$ patients. Similarly, low NLR group (LNLR <115), 30 patients and the high PLR group (HPLR $\geq 115), 66$ patients were established.

\subsection{Association of NLR and PLR with clinical parameters}

In order to investigate the relationship of NLR and PLR with the clinical parameters in osteosarcoma patients, the comparisons between LNLR(LPLR) and HNLR(HPLR) have been conducted (Table 1). Our results demonstrated that the tumor size in patients with low NLR was significantly smaller than that with high NLR $\left(171.37 \pm 12.94 \mathrm{~cm}^{3}\right.$ vs $\left.218.34 \pm 11.24 \mathrm{~cm}^{3}, p=0.034\right)$. The similar result could be found in PLR group $\left(173.22 \pm 10.54 \mathrm{~cm}^{3}\right.$ vs $\left.201.97 \pm 15.24 \mathrm{~cm}^{3}, \mathrm{p}=0.041\right)$. Additionally, patients with advanced Enneking stage and elevated ALP was significantly associated with high NLR, while the rate of these two parameters was no significant difference in PLR group. 
Table 1

Association between clinical data and NLR/PLR

\begin{tabular}{|c|c|c|c|c|c|c|c|}
\hline \multirow[t]{2}{*}{ clinical parameters } & \multirow[t]{2}{*}{$\mathbf{n}$} & \multicolumn{3}{|l|}{ NLR } & \multicolumn{3}{|l|}{ PLR } \\
\hline & & $\begin{array}{l}\text { LNLR } \\
(n=50)\end{array}$ & $\begin{array}{l}\text { HNLR } \\
(n=46)\end{array}$ & $\begin{array}{l}p- \\
\text { value }\end{array}$ & $\begin{array}{l}\text { LPLR } \\
(n=30)\end{array}$ & $\begin{array}{l}\text { HPLR } \\
(n=66)\end{array}$ & $\begin{array}{l}p- \\
\text { value }\end{array}$ \\
\hline Age (year) & & & & 0.447 & & & 0.823 \\
\hline$\leq 17$ & 56 & 31 & 25 & & 18 & 38 & \\
\hline$>17$ & 40 & 19 & 21 & & 12 & 28 & \\
\hline Sex & & & & 0.959 & & & 0.165 \\
\hline Male & 54 & 28 & 26 & & 20 & 34 & \\
\hline Female & 42 & 22 & 20 & & 10 & 32 & \\
\hline Tumor location & & & & 0.949 & & & 0.363 \\
\hline Tibia & 40 & 28 & 12 & & 16 & 24 & \\
\hline Femur & 44 & 30 & 14 & & 10 & 34 & \\
\hline Others & 12 & 8 & 4 & & 4 & 8 & \\
\hline Tumor size $\left(\mathrm{cm}^{3}\right)$ & $\begin{array}{l}195.37 \\
\pm 8.74\end{array}$ & $\begin{array}{l}171.37 \pm \\
12.94\end{array}$ & $\begin{array}{l}218.34 \pm \\
11.24\end{array}$ & 0.034 & $\begin{array}{l}173.22 \pm \\
10.54\end{array}$ & $\begin{array}{l}201.97 \pm \\
15.24\end{array}$ & 0.041 \\
\hline Enneking stage & & & & 0.024 & & & 0.321 \\
\hline$\square$ & 28 & 18 & 10 & & 10 & 18 & \\
\hline$\square$ & 46 & 26 & 20 & & 16 & 30 & \\
\hline$\square$ & 22 & 6 & 16 & & 4 & 18 & \\
\hline Subtype & & & & 0.202 & & & 0.362 \\
\hline Osteoblastic & 58 & 34 & 24 & & 15 & 43 & \\
\hline Chondroblastic & 22 & 8 & 14 & & 9 & 13 & \\
\hline Others & 16 & 8 & 8 & & 6 & 10 & \\
\hline ALP & & & & 0.013 & & & 0.763 \\
\hline Elevated & 50 & 20 & 30 & & 16 & 34 & \\
\hline Normal & 46 & 30 & 16 & & 14 & 32 & \\
\hline ESR & & & & 0.478 & & & 0.352 \\
\hline Elevated & 70 & 38 & 32 & & 20 & 50 & \\
\hline
\end{tabular}




\begin{tabular}{|c|c|c|c|c|c|c|c|}
\hline \multirow[t]{2}{*}{ clinical parameters } & \multirow[t]{2}{*}{$\mathbf{n}$} & \multicolumn{3}{|l|}{ NLR } & \multicolumn{3}{|l|}{ PLR } \\
\hline & & $\begin{array}{l}\text { LNLR } \\
(n=50)\end{array}$ & $\begin{array}{l}\text { HNLR } \\
(n=46)\end{array}$ & $\begin{array}{l}p- \\
\text { value }\end{array}$ & $\begin{array}{l}\text { LPLR } \\
(n=30)\end{array}$ & $\begin{array}{l}\text { HPLR } \\
(n=66)\end{array}$ & $\begin{array}{l}p- \\
\text { value }\end{array}$ \\
\hline Normal & 26 & 12 & 14 & & 10 & 16 & \\
\hline CRP & & & & 0.148 & & & 0.161 \\
\hline Elevated & 64 & 38 & 26 & & 17 & 47 & \\
\hline Normal & 32 & 14 & 18 & & 13 & 19 & \\
\hline Mononuclear $\left(10^{9} / \mathrm{L}\right)$ & $\begin{array}{l}0.64 \pm \\
0.28\end{array}$ & $\begin{array}{l}0.58 \pm \\
0.23\end{array}$ & $\begin{array}{l}0.66 \pm \\
0.29\end{array}$ & 0.376 & $\begin{array}{l}0.64 \pm \\
0.20\end{array}$ & $\begin{array}{l}0.65 \pm \\
0.31\end{array}$ & 0.782 \\
\hline
\end{tabular}

\subsection{Relationship between clinical parameters and PCR in univariate and multivariate analysis}

There were 36 patients (37.5\%) possess a pCR after NACT of osteosarcoma in totally. Univariate analysis was conducted to determine the risk factor that influenced PCR (Table 2). Our result revealed that the smaller tumor volum, lower stage, lower NLR and lower PLR were more likely to achieve pCR.

Subsequently, Tumor size, Enneking stage, NLR and PLR were included in multivariate analyses, which further confirmed that NLR before treatment was an independent risk factor for pCR. Compared to patients with a high NLR, those with a low NLR showed more than 2-fold higher chance to achieve pCR (OR 2.82, 95\% Cl 1.36-5.17, $\mathrm{p}=0.02$ ). 
Table 2

Association between patient/tumor data and pCR in univariate

\begin{tabular}{|llll|}
\hline Variable & $\mathbf{n}$ & pCR(\%) & p value \\
\hline Age (year) & & & 0.669 \\
$\leq 17$ & 56 & $22(39.3)$ & \\
$>17$ & 40 & $14(35.0)$ & \\
\hline Sex & & & 0.111 \\
\hline Male & 54 & $24(44.4)$ & \\
\hline Female & 42 & $12(28.6)$ & \\
\hline Tumor location & & & 0.896 \\
\hline Tibia & 40 & $16(40.0)$ & \\
\hline Femur & 44 & $16(36.4)$ & \\
\hline Others & 12 & $4(33.3)$ & \\
\hline Tumor size(cm ${ }^{3}$ ) & & & 0.034 \\
\hline$\leq 195.37$ & 38 & $20(52.6)$ & \\
\hline$>195.37$ & 58 & $18(31.0)$ & \\
\hline Enneking stage & & & 0.023 \\
\hline$\square$ & 28 & $15(53.6)$ & \\
\hline$\square$ & 46 & $16(34.8)$ & \\
\hline$\square$ & 22 & $5(22.7)$ & \\
\hline Subtype & & & \\
\hline Osteoblastic & 58 & $23(39.7)$ & \\
\hline Chondroblastic & 22 & $6(27.3)$ & \\
\hline Others & 16 & $7(43.8)$ & \\
\hline ALP & & & \\
\hline Elevated & 50 & $21(42.0)$ & \\
\hline Normal & & & \\
\hline ESR & & & \\
\hline Elevated & & & \\
\hline
\end{tabular}




\begin{tabular}{|llll|}
\hline Variable & $\mathbf{n}$ & $\mathbf{p C R}(\%)$ & p value \\
\hline Normal & 26 & $8(30.8)$ & \\
CRP & & & 0.592 \\
\hline Elevated & 64 & $25(39.1)$ & \\
Normal & 32 & $11(34.4)$ & \\
NLR & & & 0.021 \\
LNLR & 50 & $22(44.0)$ & \\
HNLR & 46 & $14(30.4)$ & \\
PLR & & & 0.031 \\
\hline LPLR & 30 & $16(53.3)$ & \\
HPLR & 66 & $20(30.3)$ & \\
\hline
\end{tabular}

Table 3

Association between patient/tumor characteristics and pCR in multivariate analysis

\begin{tabular}{|llll|}
\hline Variable & OR & $\mathbf{9 5 \%} \mathrm{Cl}$ & P value \\
\hline Tumor size $\leq 195.37$ vs $>195.37$ & 1.43 & $0.29-6.88$ & 0.65 \\
\hline Grading G1 vs G2/G3 & 0.98 & $0.31-3.21$ & 0.98 \\
\hline LNLR vs HNLR & 2.82 & $1.36-5.17$ & 0.02 \\
\hline LPLR vs HPLR & 0.73 & $0.10-5.41$ & 0.76 \\
\hline
\end{tabular}

\section{Discussion}

In this multicenter study, we provided the first assessment cocerning on the predictive value of NLR to the effectiveness of NACT in patients with osteosarcoma. Ninety-six patients who were going to treatment of NACT were included and NLR and PLR were calculated. We found that patients with low pretreatment NLR (<2.36) were significantly associated with higher rate of pCR and better effect of NACT. However, other clinical parameters, including age, sex, tumor location, size, clinical stage, tumour subtype, ALP, ESR, CRP and PLR presented no relevance with $\mathrm{PCR}$ in our study.

Recently, there were several studies demonstrated that immune system plays a key role in tumor response to chemotherapy. In a recent study by Chae and his colleague [15], the data of 87 patients with breast cancer was retrospectively analyzed. They found that patients in the low NLR group had a higher pCR compared to those in the high NLR group $(42.1 \%$ vs $18.4 \%, p=0.018,95 \% \mathrm{Cl}: 1.36-5.17)$. Kim et al. [8] 
used pre-treatment NLR and PLR as prognostic indicators of pCR in patients with gastric cancer. Our observations are consistent with those predecessors.

The association between an elevated NLR and pCR is complex. Studies have shown that inflammation not only induces tumorigenesis, but also promotes tumor metastasis and drug resistance. Firstly, inflammatory cells in tumor micro-environment can secrete a variety of cytokines, chemokines and cytotoxic mediators, which can induce early cell carcinogenesis and promote tumor occurrence [16]. Secondly, they can activate a variety of downstream transcription factors, induce the expression of antiapoptotic genes and activate cyclin, thus promoting the survival and proliferation of tumor cells [17]. What's more, inflammatory reaction can activate a variety of enzymes, which can make tumor cells more aggressive and metastatic by degrading extracellular matrix ${ }^{18}$. Neutrophils and lymphocytes are the most important cells of inflammatory reaction, and they are the bridge of inflammation in tumor microenvironment. Neutrophils can promote the reconstruction of extracellular matrix and promote tumor growth, metastasis and drug resistance [19-21] In addition, neutrophils promote angiogenesis by releasing vascular endothelial factors, thus promoting tumor invasion. In addition, neutrophils promote angiogenesis by releasing vascular endothelial growth factor (VEGF), thus promoting tumor invasion [22]. On the contrary, lymphocyte mediated cytotoxicity can inhibit tumor proliferation and metastasis [23]. In general, the higher the NLR, the more severe the systemic inflammatory response, suggesting that the tumor has stronger invasiveness, higher malignancy and stronger drug resistance. Therefore, the higher the NLR, the worse the effect of NACT, and the lower the incidence of pCR.

\section{Conclusions}

Our findings suggest that NLR is an important factor predicting the response to NACT in osteosarcoma patients. Patients with higher NLR showed a lower percentage of pCR after NACT.

\section{Declarations}

\section{Acknowledgments}

The authors would like to thank all the staff of the participating departments.

\section{Authors' contributions}

THJ, LY and LDH designed the study. JSY, LSJ and LJL collected the data. LSL and WQJ evaluated the data. THJ and LSL wrote the manuscript. All authors read and approved the final manuscript.

\section{Funding}

This study has been supported by the Fund for National Natural Science Foundation of China (No. 81960768), Natural Science Foundation of Guangxi (No.2019JJA140408), Youth Science and Technology Project of the First Affiliated Hospital of Guangxi Medical University (No. 201903038), and 
Medical Excellence Award" Funded by the Creative Research Development Grant from the First Affiliated Hospital of Guangxi Medical University.

\section{Availability of data and materials}

All the data needed to achieve the conclusion are presented in the paper.

\section{Ethics approval and consent to participate}

The study was approved by the Ethics Committee of Minzu Affiliated Hospital of Guangxi Medical University and the First Affiliated Hospital of Guangxi Medical University. The authors declare that all investigations were conducted in conformity with ethical standards.

\section{Consent for publication}

We obtained written consent from the patient of the participant before publishing this information.

\section{Competing interests}

The authors declare that they have no competing interests.

\section{Author details}

${ }^{1}$ Departments of Orthopedics, Minzu Affiliated Hospital of Guangxi Medical University, Nanning, Guangxi, China

${ }^{2}$ People's Hospital of Baise, Baise, Guangxi, China

${ }^{3}$ Departments of Surgery of bone and soft tissue tumor, Affiliated Tumor Hospital of Guangxi Medical University, Nanning, Guangxi, China

${ }^{4}$ Departments of General Practice, The First Affiliated Hospital of Guangxi Medical University, Nanning, Guangxi, China

${ }^{5}$ Departments of Orthopedics, The First Affiliated Hospital of Guangxi Medical University, Nanning, Guangxi, China

${ }^{6}$ Department of Spine and Osteopathic Surgery, The First Affiliated Hospital of Guangxi Medical University, Nanning, Guangxi, China

\section{References}

1. Bielack S, Carrle D, Casali PG. Osteosarcoma: ESMO clinical recommendations for diagnosis, treatment and follow-up. Ann Oncol. 2009;20 Suppl 4:137-9. doi:10.1093/annonc/mdp154. 
2. Gross SW. Sarcomas of the long bones: based on a study of one hundred and sixty five cases. Am J Med Sci. 1879, 8: 338-377.

3. Cortes EP, Holland JF,Wang JJ, et al. Adriamycin and amputation in primary osteogenic sarcoma. Proceedings of the American Association for Cancer Research Annual Meeting. 1974, 15(3):170.

4. Rosen G, Suwansirikul S, Kwon C, et al. High dose methotrexate with citrovorum factor rescue and adriamycin in childhood osteogenic sarcoma. Cancer. 1974, 33(4):1151-1161.

5. Faisham W I, Mat Saad A Z, Alsaigh L N, et al. Prognostic factors and survival rate of osteosarcoma: A single-institution study. Asia-Pacific Journal of Clinical Oncology. 2017, 13(2):e104-e110.

6. National Comprehensive Cancer Network.Bone Cancer (Version 1.2018). Accessed August 29, 2017.

7. Chen $\mathrm{Y}$, Chen $\mathrm{K}$, Xiao X, et al. Pretreatment neutrophil-to lymphocyte ratio is correlated with response to neoadjuvant chemotherapy as an independent prognostic indicator in breast cancer patients: a retrospective study. BMC Cancer, 2016, 16(1):320.

8. Kim M S, Lee S Y, Cho W H, et al. Initial tumor size predicts histologic response and survival in localized osteosarcoma patients. Journal of Surgical Oncology. 2008, 97(5):456-461.

9. De Vries AF, Kremser C, Hein PA, et al. Tumor microcirculation and diffusion predict therapy outcome for primary rectal carcinoma. Int J Radiat Oncol Biol Phys, 2003, 56:958-965.

10. Balkwill F R, Mantovani A. Cancer-related inflammation: Common themes and therapeutic opportunities[J]. Seminars in Cancer Biology, 2012, 22(1):33-40.

11. DOLANRD, MCSORLEYST, HORGANPG, et al. The role of the systemic inflammatory response in predicting outcomes in patients with advanced inoperable cancer: Systematic review and meta analysis. Crit Rev Oncol Hematol. 2017, 116:134囚146.

12. Chen $\mathrm{Y}$, Chen $\mathrm{K}$, Xiao $\mathrm{X}$, et al. Pretreatment neutrophil-to-lymphocyte ratio is correlated with response to neoadjuvant chemotherapy as an independent prognostic indicator in breast cancer patients: a retrospective study. BMC Cancer, 2016, 16(1):320.

13. Mclaren P J, Bronson N W, Hart K D, et al. Neutrophil-to-Lymphocyte and Platelet-to-Lymphocyte Ratios can Predict Treatment Response to Neoadjuvant Therapy in Esophageal Cancer. Journal of Gastrointestinal Surgery, 2017, 21(4):607-613.

14. Enneking WF. Musculoskeletal tumor surgery. New York (NY): Churchil Livingstone, Inc; 1983.

15. Chae, S., et al., Neutrophil-lymphocyte ratio predicts response to chemotherapy in triple-negative breast cancer. Current Oncology, 2018. 25(2): p. e113.

16. P, A., et al., Pathways connecting inflammation and cancer. Current opinion in genetics \& development, 2008. 18(1): p. 3-10.

17. F, O., Inflammation-related carcinogenesis: current findings in epidemiological trends, causes and mechanisms. Yonago acta medica, 2014. 57(2): p. 65-72.

18. SI, G., et al., Intracellular signals and events activated by cytokines of the tumor necrosis factor superfamily: From simple paradigms to complex mechanisms. International review of cytology, 2006. 252(undefined): p. 129-61. 
19. JE, D.L., W. BR, and F. LT, The potential role of neutrophils in promoting the metastatic phenotype of tumors releasing interleukin-8. Clinical cancer research: an official journal of the American Association for Cancer Research, 2004. 10(15): p. 4895-900.

20. I, M., et al., Polymorphonuclear neutrophils and T lymphocytes: strange bedfellows or brothers in arms? Trends in immunology, 2009. 30(11): p. 522-30.

21. PC, R., et al., Arginase I-producing myeloid-derived suppressor cells in renal cell carcinoma.

22. CA, D., L. S, and B. S, Modulation of neutrophil granulocytes in the tumor microenvironment: mechanisms and consequences for tumor progression. Seminars in cancer biology, 2013. 23(3): p. 141-8.

23. HE, O., et al., Peripheral lymphocyte and eosinophil counts as indicators of prognosis in primary breast cancer. Cancer, 1983. 52(1): p. 126-30.

\section{Figures}




\section{ROC Curve}

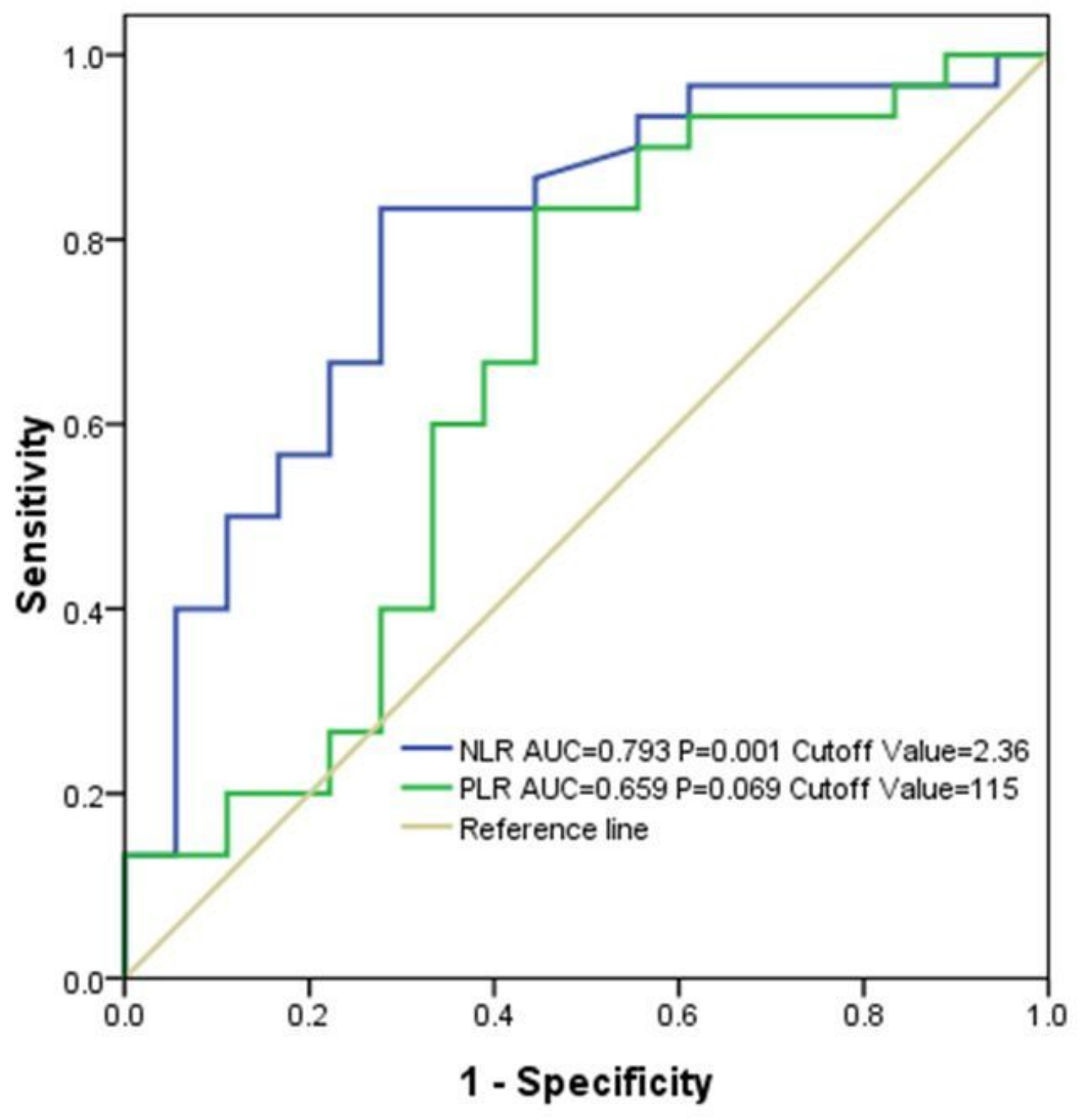

Figure 1

As Figure 1 shows, when pCR was defined as an end point, the areas under the curve (AUC) for NLR and PLR were $0.793(p=0.001)$ and $0.659(p=0.069)$, respectively. 